\title{
High Security Chipless RFID Tags Using Frequency Shift Coding Technique
}

\author{
M. SUMI ${ }^{1}$, Anju PRADEEP ${ }^{2}$, Binu PAUL ${ }^{2}$, S. MRIDULA ${ }^{2}$ \\ ${ }^{1}$ Dept. of Electronics and Communication, N S S College of Engineering, Palakkad, Kerala, India \\ ${ }^{2}$ School of Engineering, Cochin University of Science and Technology, Cochin- 22, Kerala, India \\ sumi.harikrishnan@gmail.com
}

Submitted December 24, 2016 / Accepted April 17, 2017

\begin{abstract}
A high security chipless RFID tag designed using $E$ shaped resonator is presented in this paper. The tag identity is encoded using Frequency Shift Coding technique. 144 different code words are possible in 2.78 to $3.85 \mathrm{GHz}$ band using two E shaped resonators. The tag identity can be decoded from either amplitude or group delay information. The resonators are designed and fabricated on substrate C-MET LK4.3 of dielectric constant 4.3 and loss tangent 0.0018. Different tag combinations are designed and tested using bistatic measurement setup. Measurement results on realized prototypes are provided to ensure the reliability of the proposed design.
\end{abstract}

\section{Keywords}

Bistatic measurement, chipless RFID tag, E shaped resonator, frequency shift coding technique, group delay

\section{Introduction}

Radio frequency identification (RFID) is an electronic tagging technology which utilizes radio frequency waves to remotely detect and identify a device or an object using RFID system. The RFID system consists of two main elements : RFID tag, where data is encoded, and the RFID reader, which is used for extracting the encoded data from the tag. The tags are typically chip based and generally contain silicon chips and antennas. RFID tags can be classified based on different attributes like sources of power for operation, frequencies used etc. Based on the method by which the tags are powered, the tags can be classified as active tags and passive tags. RFID tag with onboard power source and associated electronics for performing specialized tasks is called active tag. It can be designed with a variety of specialized electronic devices, including microprocessors, different types of sensors, or Input and Output devices etc. Passive tag has neither onboard power source nor an active transmitter. Here, the electromagnetic signal transmitted by the RFID reader inductively powers the tag, which helps it to retransmit its information. Depending on the frequency of operation, RIFD tags are classified as low frequency $(120 \mathrm{KHz}-150 \mathrm{KHz})$, high frequency (13.56 MHz), ultra high frequency $866 \mathrm{MHz}$ - $868 \mathrm{MHz}$ (European Union),902 MHz - $928 \mathrm{MHz}$ (North American continent) and microwave bands $(2.45 \mathrm{GHz}$ and $5.8 \mathrm{GHz}$ ) [1]. RFID serves as a radio technology to achieve internet of things (IoT) vision to create a worldwide network of smart objects [2]. If all objects are equipped with radio tags, they could be identified and inventoried. To fulfil these functions, RFID tags must be data dense, inexpensive and energy efficient [3]. However, their widespread use is limited due to the high cost of silicon chips and power requirement. Hence, cost effective solutions are of great need. Today, RFID systems are being developed with tags that do not contain silicon chips. These tags are known as chipless tags. When multiple chipless tags are in the interrogation zone of a reader, anticollision algorithms needs to be employed for correct tag identification.

Compared to conventional tags containing silicon chip, chipless RFID tag uses an entirely different technique to encode data. Each chipless RFID tag contains a planar passive circuit. This planar circuit will reflect back a unique electromagnetic signal to the reader. The tag identity is decoded from this reflected signal. Different approaches for designing spectral signature based chipless tags are reported in [4-11]. In these tags, each data bit is identified as the absence or presence of a resonance at a predetermined frequency. Majority of the tags are designed on microstrip based resonators. Capacitively tuned dipole for RFID barcode was first reported by Jalaly and Robertson [4]. Here the tag consists of arrays of identical microstrip dipoles capacitively tuned to different resonant frequencies. Space-filling curves using fractal geometries such as Hilbert and Peano curves to generate the frequency signature from backscattered resonances are reported in [5]. To encode the data, space-filling curves need significant layout modifications while capacitively tuned dipoles have undesired parasitic effects and size limitations. Chipless RFID tag using meander complementary split ring resonator is proposed in [6]. The tag requires a frequency band of $4 \mathrm{GHz}$ to $7 \mathrm{GHz}$ to encode four bits. An eight bit chipless RFID tag using quarter wave open stub resonator is reported in [7], [8]. The tag encodes eight bits in a frequency band from $2.08 \mathrm{GHz}$ to $4.03 \mathrm{GHz}$. A two bit planar chipless RFID 
tag based on group delay encoding is presented in [9]. This tag requires very large area for encoding more number of bits. Chipless RFID tag using coplanar waveguide fed circular monopole antennas and multiresonators is reported in [10]. It reports a multiresonator which requires a frequency band of $6 \mathrm{GHz}$ to $8 \mathrm{GHz}$ to encode seven bits. A dual-polarized directly printable moisture sensor chipless RFID tag for intelligent packaging application is reported in [11]. It reports a 24 bit tag having a bandwidth of $13.5 \mathrm{GHz}$.

Most of these designs use absence or presence coding technique to encode the tag identity. In absence or presence coding technique, maximum bits that can be represented by an RFID tag is limited by the number of resonators. The bit encoding capacity of the tag can be enhanced using Frequency Shift Coding (FSC) technique, by encoding more than one bit per resonator [12]. This method is more appropriate in the case where large data encoding is required with less number of resonators. Another advantage is the design of high security tags, ie; the tags appear to be similar, but the identification code will be different.

This paper focuses on the design of chipless tags using FSC technique with high data encoding capacity and design flexibility for high security applications. The tag identity can be decoded either from amplitude or group delay information.

\section{Resonator Design}

The resonator structure has evolved from a single $\mathrm{E}$ shaped structure. The performance of E shaped resonator is evaluated in this section to justify its candidature for RFID applications. E shaped resonator excited by a $50 \Omega$ microstrip transmission line is shown in Fig. 1(a). The prototype is simulated on the substrate C-MET/LK4.3 with dielectric constant 4.3, height $1.6 \mathrm{~mm}$ and loss tangent 0.0018 using CST MWS. The simulated transmission characteristics of E shaped resonator is shown in Fig. 1(b). The design approach is to generate multiple resonances by modifying the middle arm $\left(L_{i}\right)$ of E shaped resonator.

The resonant frequency of the E shaped resonator can be found by the relation

$$
f=\frac{c}{2 .\left(L_{i}+W_{a}+L_{k}+\Delta L\right) \cdot \sqrt{\varepsilon_{\mathrm{eff}}}}
$$

where $L_{i}$ is the length of middle arm, $W_{a}$ is the distance between lower arm and middle arm, $L_{k}$ is the length of lower arm (Fig. 1(a)), $\Delta L$ is the fringing length, $\varepsilon_{\text {eff }}$ is the effective permittivity of the substrate and $\mathrm{c}$ is the velocity of light. $\lambda_{g}$ is the guide wavelength evaluated at the resonant frequency given by

$$
\lambda_{g}=\frac{\lambda}{\sqrt{\varepsilon_{\mathrm{eff}}}}
$$

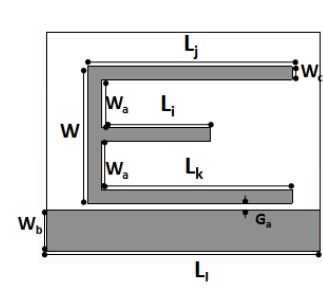

(a)

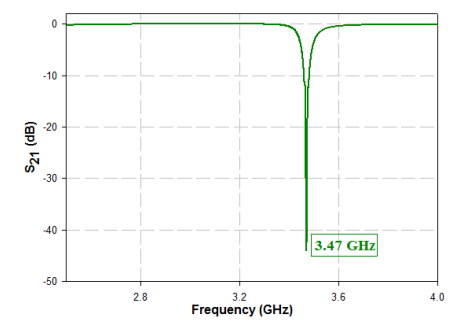

(b)
Fig. 1. (a) $E$ shaped resonator $\left[L_{j}=15, L_{i}=9.54, L_{k}=14, \mathrm{~W}\right.$ $=10, L_{l}=20, W_{a}=3.5, W_{c}=1, G_{a}=0.5, W_{b}=3$ (All dimensions in mm), Substrate: loss tangent $=0.0018, \epsilon_{r}$ $=4.3, \mathrm{~h}=1.6 \mathrm{~mm}](\mathrm{b})$ Simulated transmission characteristics of the $\mathrm{E}$ shaped resonator.

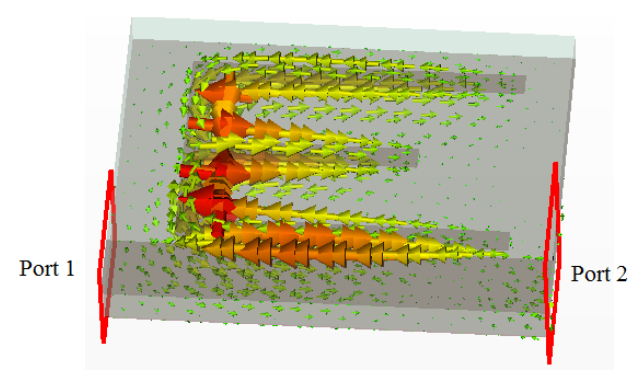

(a) $3.47 \mathrm{GHz}$

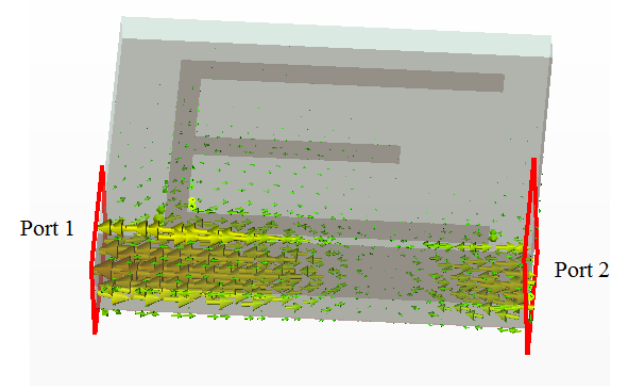

(b) $4 \mathrm{GHz}$
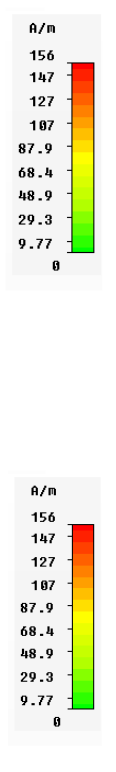

Fig. 2. Surface current distribution of $E$ shaped resonator at resonant frequency of $3.47 \mathrm{GHz}$ and at a non-resonant frequency of $4 \mathrm{GHz}$.

Surface current distribution of E shaped resonator at its resonant frequency $(3.47 \mathrm{GHz})$ and at a non-resonant frequency $(4 \mathrm{GHz})$ are depicted in Fig. 2. The surface current distribution is concentrated in the resonator at the resonant frequency. It is clear that there is no transmission from port 1 to port 2 at the resonant frequency and transmission occurs at non-resonant frequency.

\section{Frequency Shift Coding Applied to Multiresonator with Two E Shaped Resonators}

Two types of data encoding methods are widely used for Chipless RFID tag namely Absence or presence coding technique and Frequency shift coding technique. In absence or presence coding technique, one resonator represents one 


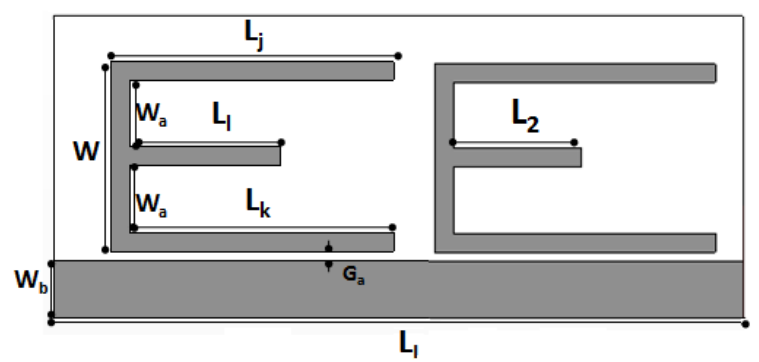

Fig. 3. Layout of multiresonator with two $E$ shaped resonators $L_{j}=15, L_{k}=14, \mathrm{~W}=10, L_{l}=40, W_{a}=3.5, G_{a}=0.5$, $W_{b}=3$ (All dimensions in $\mathrm{mm}$ ), Substrate: loss tangent $=0.0018, \epsilon_{r}=4.3, \mathrm{~h}=1.6 \mathrm{~mm}$

\begin{tabular}{|l|l|l|}
\hline $\begin{array}{l}\text { Resonant fre- } \\
\text { quency }\end{array}$ & $\Delta f_{1}(2.78-3.28)$ & $\Delta f_{2}(3.3-3.85)$ \\
\hline$f_{1}$ & 2.78 & 3.3 \\
\hline$f_{2}$ & 2.85 & 3.35 \\
\hline$f_{3}$ & 2.89 & 3.40 \\
\hline$f_{4}$ & 2.93 & 3.45 \\
\hline$f_{5}$ & 2.96 & 3.51 \\
\hline$f_{6}$ & 3.02 & 3.56 \\
\hline$f_{7}$ & 3.06 & 3.60 \\
\hline$f_{8}$ & 3.10 & 3.66 \\
\hline$f_{9}$ & 3.16 & 3.71 \\
\hline$f_{10}$ & 3.20 & 3.75 \\
\hline$f_{11}$ & 3.80 \\
\hline$f_{12}$ & 3.24 & 3.85 \\
\hline
\end{tabular}

Tab. 1. $\Delta f$ and $f$ of multiresonator with two E shaped resonators (All values in $\mathrm{GHz}$ ).

bit of information. The presence of resonance at a particular frequency is used to encode a logic 1 and the absence of resonance is used to encode a logic 0 . So with $\mathrm{n}$ resonators only $2^{n}$ combinations are possible. FSC Technique is utilized to enhance the bit coding capacity of the tag [12], [13]. Here resonators are assigned with different frequency band $(\Delta \mathrm{f})$ and each frequency band is divided into different resolution bandwidth $(\delta \mathrm{f}) . \Delta \mathrm{f}$ can be selected depending on the available frequency spectrum and the number of resonators. $\delta \mathrm{f}$ is the required frequency band for each resonator to successfully represent its resonant frequency. Therefore, one resonator can represent more number of states. So this technique allows an enhancement in coding efficiency, by encoding more than one bit per resonator. Moreover the tags appear to be similar, since there is only a slight variation in middle arm length of E shaped resonator from tag to tag, but the identification code will be different. This helps in design of high security tags.

Figure 3 shows the layout of multiresonator with two E shaped resonators whose length $\left(L_{i}\right)$ is individually varied for FSC. $\Delta \mathrm{f}$ and resonant frequency $(f)$ of each resonator are listed in Tab. 1.

Variation of the middle arm length of the first resonator, keeping the dimensions of the second resonator constant, results in twelve different resonant frequencies as shown in Fig. 4. Similarly, variation of the middle arm length of the second resonator, keeping the dimensions of the first resonator constant, results in twelve different resonant frequencies as shown in Fig. 5.

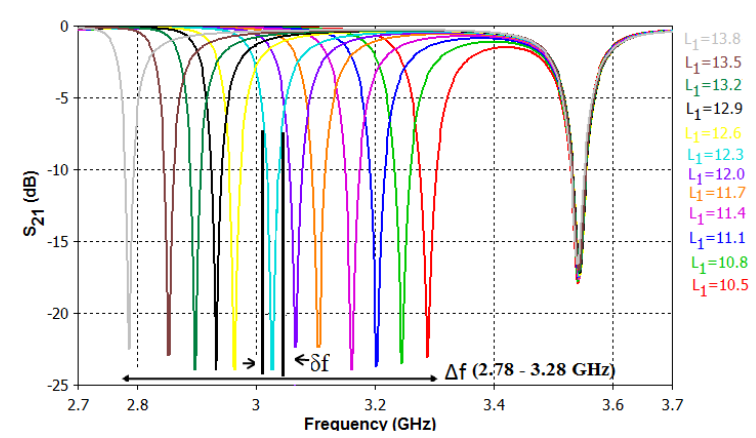

(a) $S_{21}$

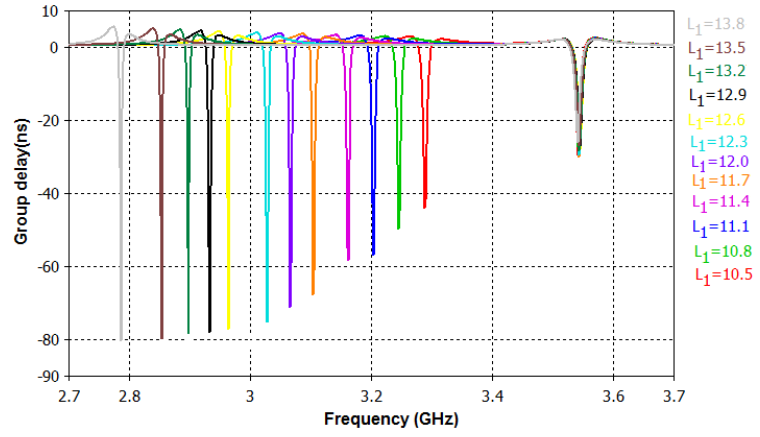

(b) Group delay

Fig. 4. Simulated transmission characteristics of tuning the first E shaped resonator in multiresonator with two E shaped resonators shown in Fig. 3 ( $L_{2}=9 \mathrm{~mm}, L_{1}$ in $\left.\mathrm{mm}\right)$.

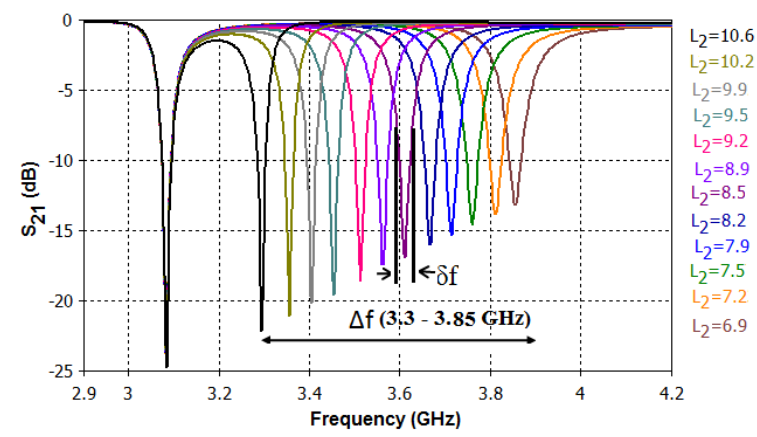

(a) $S_{21}$

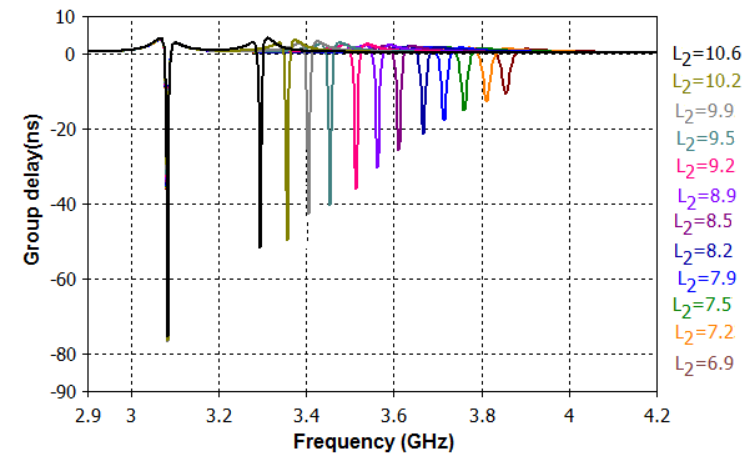

(b) Group delay

Fig. 5. Simulated transmission characteristics of tuning the second $\mathrm{E}$ shaped resonator in multiresonator with two $\mathrm{E}$ shaped resonators shown in Fig. $3\left(L_{1}=12 \mathrm{~mm}, L_{2}\right.$ in $\mathrm{mm})$. 


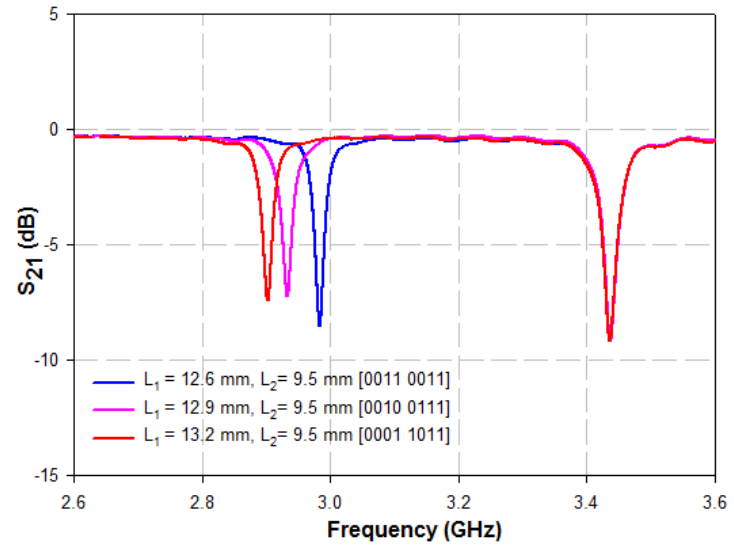

(a) $S_{21}$

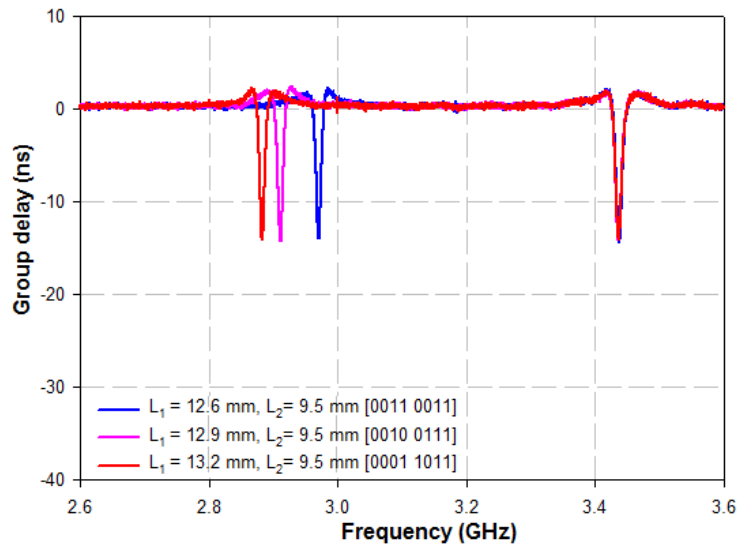

(b) Group delay

Fig. 6. Measured transmission characteristics of the multiresonator with two E shaped resonators for three different configurations by varying the length of middle arm of first $\mathrm{E}$.

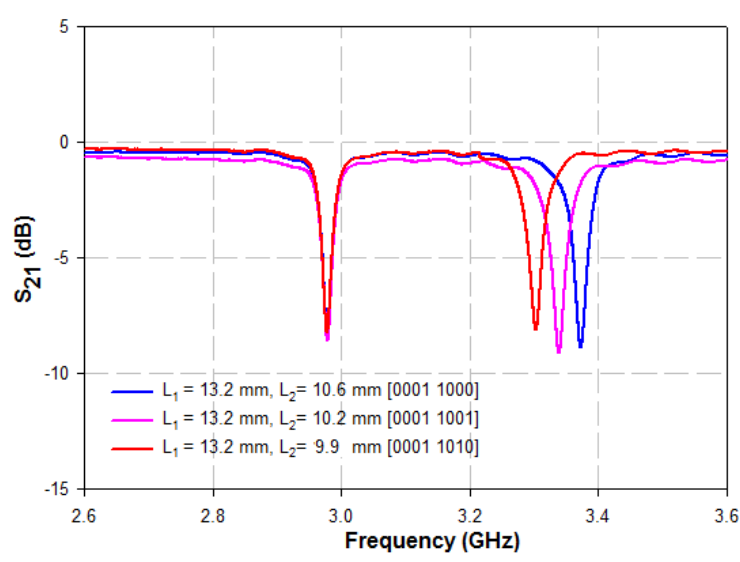

(a) $S_{21}$

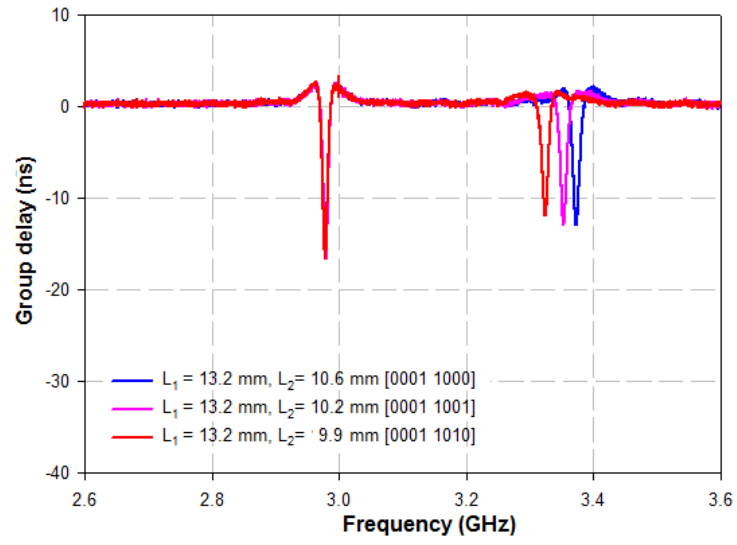

(b) Group delay

Fig. 7. Measured transmission characteristics of the multiresonator with two $\mathrm{E}$ shaped resonators for three different configurations by varying the length of middle arm of second $\mathrm{E}$.

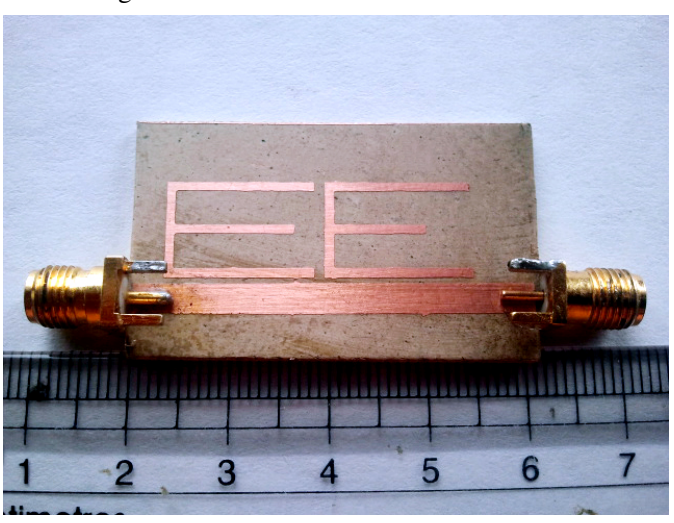

Fig. 8. Photograph of fabricated multiresonator with two E shaped resonators.

While varying the length of each resonator care must be taken that the resonance due to other resonators remain unaffected. In FSC for bit encoding, precautions must also be taken so that merging of $\Delta \mathrm{f}$ does not occur and harmonics of the lower frequency resonators do not interfere with the resonance of higher frequency resonators. For a particular value of resonance of first resonator twelve different resonances are possible for the second resonator and vice versa.
Unique identification code can be assigned to each combination of resonant frequencies. Since twelve different resonant frequencies are possible for each resonator, combining the two, 144 different codes ranging from [0000 0000] to [1000 1111] are possible with two resonators.

\section{Experimental Results}

\subsection{Experimental Results of Multiresonator}

Figure 8 shows the photograph of fabricated multiresonator with two E shaped resonators. Measurements are conducted using the PNA E8362B Vector network analyser. Figure 6 shows the measured magnitude and group delay of the multiresonator when middle arm length of second $\mathrm{E}$ shaped resonator is kept constant at $9.5 \mathrm{~mm}$ and three different values for middle arm length of first E such as $12.6 \mathrm{~mm}$, $12.9 \mathrm{~mm}$ and $13.2 \mathrm{~mm}$ is chosen corresponding to the code words 00110011,00100111 and 00011011 respectively. Similarly Fig. 7 shows the measured magnitude and group delay of the multiresonator when middle arm length of first $\mathrm{E}$ shaped resonator is kept constant at $13.2 \mathrm{~mm}$ and three different values of middle arm length of second $E$ such as $10.6 \mathrm{~mm}$, 


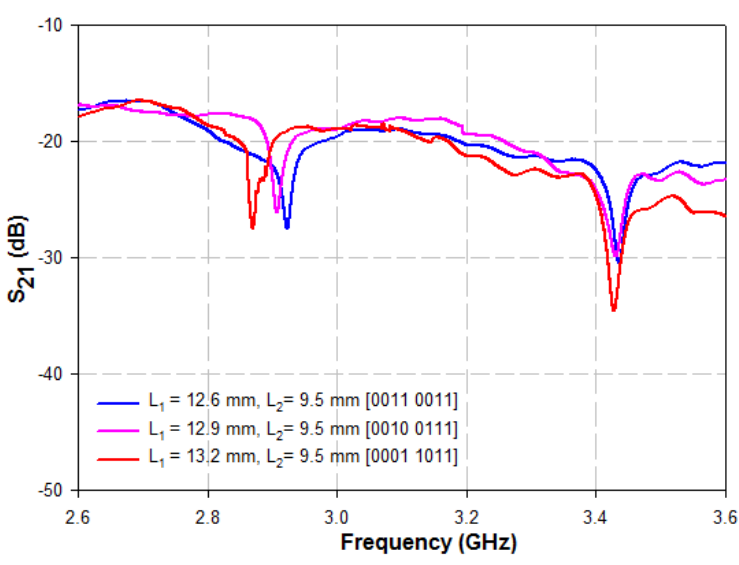

(a) $S_{21}$

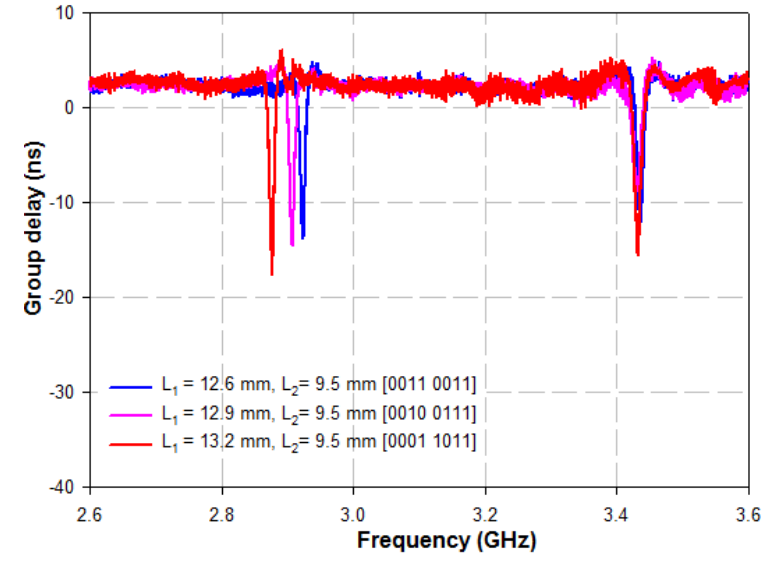

(b) Group delay

Fig. 9. Measured bistatic response of the multiresonator with two $\mathrm{E}$ shaped resonators for three different configurations by varying the length of middle arm of first $\mathrm{E}$.

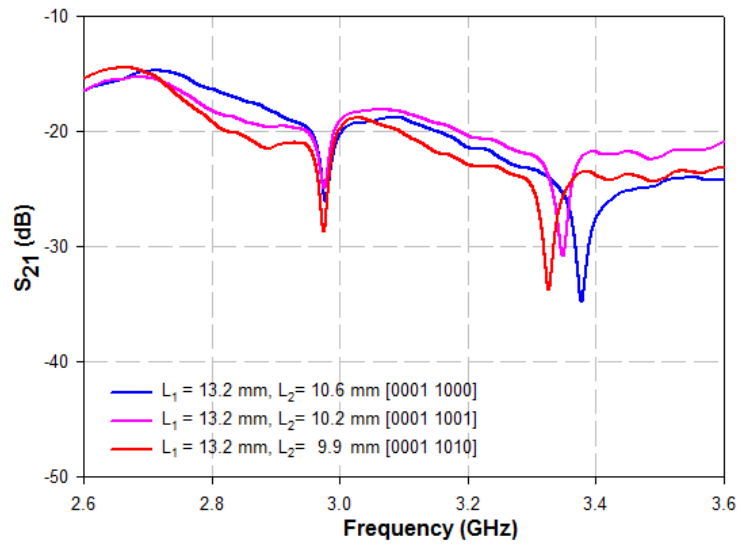

(a) $S_{21}$

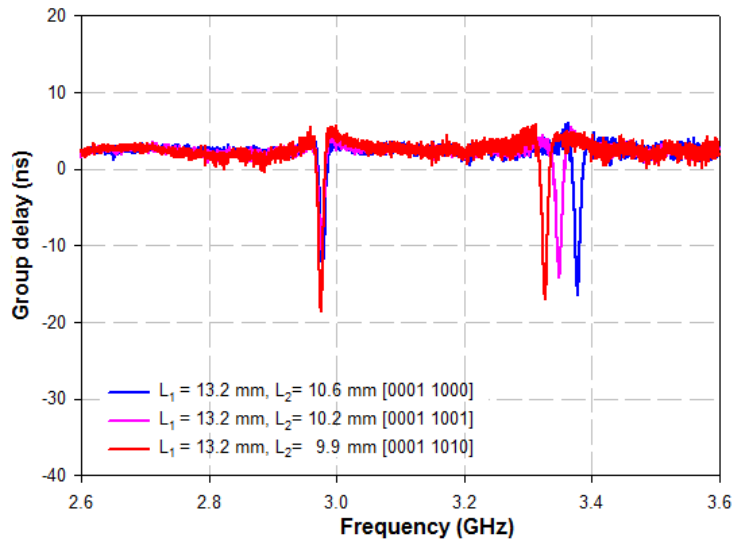

(b) Group delay

Fig. 10. Measured bistatic response of the multiresonator with two $E$ shaped resonators for three different configurations by varying the length of middle arm of second $\mathrm{E}$.

$10.2 \mathrm{~mm} 9.9 \mathrm{~mm}$ is chosen corresponding to the code words 0001 1000, 00011001 and 00011010 respectively.

\subsection{Bistatic Measurement Setup}

The proposed RFID tag incorporates two wide band antennas, one for receiving the interrogation signal from reader and the other for retransmitting the encoded signal from the multiresonator to the reader, thereby increasing the read range [14]. Disc monopole antenna is chosen due to its large frequency bandwidth. Figure 11 shows the geometry and measured reflection characteristics of the disc monopole antenna.

Two linearly polarized medium gain $(10 \mathrm{~dB})$ reader horn antennas are used for transmission of the continuous wave interrogation signal and reception of the retransmitted signal. The block schematic for the bistatic measurement is shown in Fig. 10. To provide isolation between the transmitting and receiving signals, the transmitting and receiving antennas are cross polarized. The tag is placed $20 \mathrm{~cm}$ away from horn antennas. Figures 9 and 10 show the measured bistatic response for three different configurations by varying the length of middle arm of first E shaped resonator and second E shaped resonator respectively. Figure 9 shows the measured bistatic response for the bit combinations 00110011 , 00100111 and 0001 1011. Figure 10 show the measured bistatic response for the bit combinations 00011000,0001 1001 and 00011010.

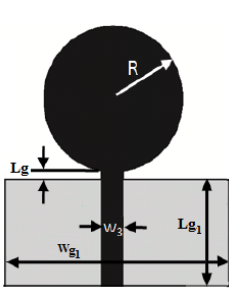

(a)

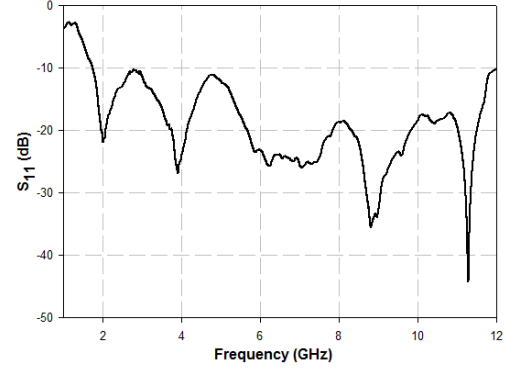

(b)
Fig. 11. (a) Disc monopole antenna $\left[\mathrm{R}=15, W_{3}=3, L_{g}=0.6\right.$, $W_{g 1}=40$ and $L_{g 1}=20$ (All dimensions in $\mathrm{mm}$ ), $\epsilon_{r}$ $=4.3$, loss tangent $=0.02, \mathrm{~h}=1.6 \mathrm{~mm}$ ] (b) Measured reflection characteristics of disc monopole antenna. 


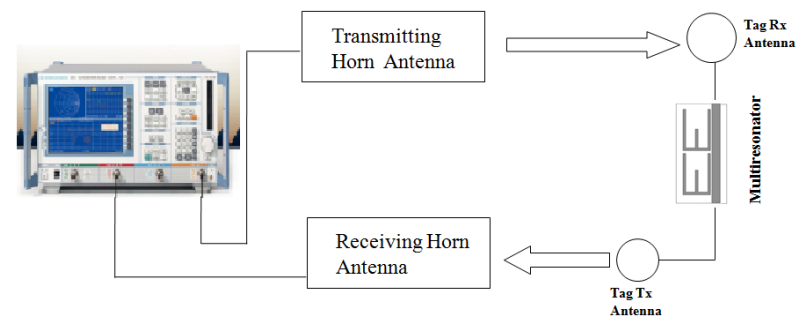

Fig. 12. Block schematic for the bistatic measurement.

\section{Conclusion}

In this paper a high security chipless RFID tag using E shaped resonator is presented. Frequency shift coding technique is used to encode the tag identity. The concept is validated from the measurements using bistatic approach. The tag can encode data in magnitude and group delay. The bit encoding capacity can be further increased by adding more E shaped resonators to the multiresonator. The cost of the tag can be reduced if implemented using low cost substrate materials.

\section{Acknowledgments}

The authors would like to thank Dr Dinesh R and Dr Nijas C M for their valuable suggestions. The authors acknowledge Dr. R. Ratheesh Scientist, C-MET, Trichur for providing the low loss C-MET LK4.3 substrate. The research is financially supported vide KSCSTE-ETP Project (ETP/03/2013/KSCSTE dated 26.09.2013).

\section{References}

[1] JANKOWSKI, P., WEGLARSKI, M., PITERA, G., et al. Development board of the autonomous semi-passive RFID transponder. Bulletin of the Polish Academy of Sciences Technical Sciences, 2016, vol. 64, no. 3, p. 647-54. DOI: 10.1515/bpasts-2016-0073

[2] BOLIC, M., ROSTAMIAN, M., DJURIC, P. M. Proximity detection with RFID: A step toward the internet of things. IEEE Pervasive Computing, 2015, vol. 14, no. 2, p. 70-76. DOI:10.1109/MPRV.2015.39

[3] NOOR, T., HABIB, A., AMIN, Y., et al. High-density chipless RFID tag for temperature sensing. Electronics Letters, 2016, vol. 52, no. 8, p. $620-622$. DOI: $10.1049 / \mathrm{el} .2015 .4488$

[4] JALALY, I., ROBERTSON, I. D. RF barcodes using multiple frequency bands. In Proceedings of the IEEE MTT-S International Microwave Symposium Digest. 2005, vol. 4, p. 1-4. DOI: 10.1109/MWSYM.2005.1516542

[5] MCVAY, J., HOORFAR, A., ENGHETA, N. Space-filling curve RFID tags. In Proceedings of the IEEE Radio and Wireless Symposium. San Diego, CA (USA), 2006, p. 199-202. DOI: $10.1109 /$ RWS.2006.1615129

[6] SAKOUHI, S., et al. A novel meander complementary split ring resonator-based RFID chipless tag. In Proceedings of the 2014 Mediterranean Microwave Symposium (MMS2014). Marrakech (Morocco), 2014, p. 1-5. DOI: 10.1109/MMS.2014.7088792
[7] NIJAS, C. M., et al. Chipless RFID tag using multiple microstrip open stub resonators. IEEE Transactions on Antennas and Propagation, 2012, vol. 60, no. 9, p. 4429-4432. DOI: 10.1109/TAP.2012.2207081

[8] NIJAS, C. M., et al. Optimisation of quarter wave microstrip open stub resonators for chipless RFID applications. In Proceedings of the IEEE Antennas and Propagation Society International Symposium (APSURSI). Orlando, FL (USA), 2013, p. 1506-1507. DOI: 10.1109/APS.2013.6711412

[9] NAIR, R., PERRET, E., TEDJINI, S. Chipless RFID based on group delay encoding. In Proceedings of the IEEE International Conference on RFID-Technologies and Applications (RFID-TA). 2011, p. 214-218. DOI: 10.1109/RFID-TA.2011.6068640

[10] ASHRAF, M. A., et al. Design of a chipless UWB RFID tag using CPW circular monopole antennas and multi-resonators. In Proceedings of the IEEE International Conference on Ubiquitous Wireless Broadband (ICUWB). Montreal (Canada), 2015, p. 1-4. DOI: 10.1109/ICUWB.2015.7324434

[11] JAVED, N., HABIB, A., AMIN, Y., et al. Directly Printable Moisture Sensor Tag for Intelligent Packaging. IEEE Sensors Journal, 2016, vol. 16, no. 16, p. 6147-6148. DOI: 10.1109/JSEN.2016.2582847

[12] VENA, A., PERRET, E., TEDJINI, S. RFID Chipless Tag Based on Multiple Phase shifters. In Proceedings of the IEEE MTT-S International Microwave Symposium Digest (MTT). 2011, p. 1-4. DOI: 10.1109/MWSYM.2011.5972712

[13] VENA, A., PERRET, E., TEDJINI, S. Design of compact and auto-compensated single-layer chipless RFID tag. IEEE Transactions on Microwave Theory and Techniques, 2012, vol. 60, no. 9, p. 2913-2924. DOI: 10.1109/TMTT.2012.2203927

[14] PRERADOVIC, S., KARMAKAR, N. C. Multiresonator-based chipless RFID system for low-cost item tracking. IEEE Transactions on Microwave Theory and Techniques, 2009, vol. 57, no. 5, p. 1411-1419. DOI: 10.1109/TMTT.2009.2017323

\section{About the Authors...}

M. SUMI received the B.Tech degree in Electronics and Communication from the Cochin University of Science and Technology (CUSAT) in 2003, and the MTech degree in Electronic Design from the Indian Institute of Science (IISc) in 2009. She is currently working towards her Ph.D. degree at School of Engineering, CUSAT. Her research interests are designing of Chipless RFIDs and multiband antennas.

Anju PRADEEP was born in India. She received the B.Tech degree in Electronics and Communication from Kerala University in 1993, the M.Tech degree in Microwave and Radar Engineering, in 2008 and $\mathrm{PhD}$ degree in 2015 from Cochin University of Science and Technology (CUSAT). She has been involved in teaching undergraduate students in Engineering colleges since 1995. She was awarded the Young Scientist Award in the field of physical sciences for the best paper presentation at the first Kerala Women's' Science Congress 2010. Her areas of interest include metamaterials and optimization techniques. She is a member of IEEE APS and a life member of the Indian Society for Technical Education. She has been working with Division of Electronics Engineering, School of Engineering, Cochin University of Science and Technology, Kerala, India since 1999. 
Binu PAUL was born in in Kerala, India in 1971. She received her $\mathrm{M}-\mathrm{Tech}$ and $\mathrm{PhD}$ degrees from Cochin University of Science and Technology, Cochin, India in 1996 and 2006 respectively. She is currently an Associate Professor of School of Engineering, CUSAT, Cochin. Her research interests include Planar antennas, Computational Electromagnetics and Compact Planar filters. She is a member of IEEE Antennas \& Propagation Society and IEEE-WIE.

S. MRIDULA was born in India. She received the BTech degree in Electronics and Communication from the Kerala University in 1988, and the MTech degree in Electronics from the Cochin University of Science and Technology (CUSAT) in 1999. She was awarded the K. G. Nair Endowment Gold Medal for securing the first rank in the university. She received her $\mathrm{PhD}$ in Microwave Electronics from CUSAT in May, 2006. She has over 23 years of teaching experience in various professional institutions in Kerala, and is presently serving as Professor, Division of Electronics Engineering, School of Engineering, CUSAT. She had won the Sessions Best Paper Award at the International Conference on Design and Modeling in Science, Education and Technology (DeMset 2011), organized by the International Institute of Informatics and Systemics at Orlando, Florida, USA, during November 29 to December 2, 2011. Her areas of interest include planar antennas, dielectric-resonator antennas, radiation hazards of mobile handset antennas, and computational electromagnetics. She is a member of various professional bodies such as IEEE, International Institute of Informatics and Systemics (IIIS), Applied Computational Electromagnetic Society, USA, a life member of the Institute of Electronics and Telecommunication Engineers (India), and a life member of the Indian Society for Technical Education. 